\title{
DIMORFISMO SEXUAL, DISTRIBUCIÓN ETARIA Y LONGEVIDAD DEL CEMENTERIO PICA 8 (PERÍODO INTERMEDIO TARDÍO, 950-1450 DC, NORTE GRANDE DE CHILE)
}

\author{
Rodrigo Retamal, ${ }^{1}$ Aryel Pacheco ${ }^{2}$ y Mauricio Uribe ${ }^{3}$
}

\section{* Introducción}

Resumen

El objetivo de este trabajo fue estudiar la distribución por sexo, edad y longevidad de los individuos adultos de la colección esqueletal cementerio Pica 8 ( 82 individuos), perteneciente al Complejo Pica

Tarapacá, Norte Grande de Chile. Respecto al sexo, se utilizaron los métodos de Buikstra y colaboradores para coxales y Ferembach y colaboradores para el cráneo, con lo que se pudo estimar el sexo de toda la muestra ( 35 masculinos; 47 femeninos). Respecto a la estimación de la edad, se utilizaron los métodos de Brooks y Suchey para la sínfisis púbica y Lovejoy para la superficie auricular del ilion.

Éstos presentaron estimaciones similares hasta los 39 años, pero difirieron posteriormente. Se discute la presencia de individuos seniles en este Complejo Cultural, ya que abre interesantes interrogantes sobre su rol cultural. En este sentido, es importante complementar la investigación, utilizando una visión desde la paleogerontología y en general, una visión a partir de la antropología de las edades, que podría contribuir más a la comprensión de este Complejo Cultural.

Palabras claves: Complejo Pica Tarapacá - estimación de sexo - estimación de la edad de muerte - longevidad.

The aim of this paper was to study the age, sex and longevity distributions of adult skeletons from Pica 8 skeletal collection $(82$ skeletons), belonging to the Pica Tarapacá Complex, Northern Chile. Regarding sex estimation, we utilized Buikstra and colleagues' method

for the os coxae bone and Ferembach and colleagues' method for cranial estimation was utilized. With these methods sex was estimated for all the sample ( 35 males, 47 females). Concerning age estimation, Brooks and Suchey method for pubic symphysis and Lovejoy method for iliac auricular surface were utilized. Both methods showed similar estimations until 39 years old, but differed beyond this age. The presence of senile individuals in this cultural complex is discussed as it opens interesting venues of research about their cultural role. In this sense, it is important to complement the research with a paleo gerontologic focus and in general, a focus from the Anthropology of Aging, which could contribute more to understanding of this cultural complex.

Key words: Pica Tarapacá Complex - sex estimation - age at death assessment - longevity.
El Complejo Cultural Pica Tarapacá ha sido definido en gran parte por los restos materiales recuperados del cementerio Pica 8 (Zlatar 1984) y escasos aportes desde la Antropología Biológica, a excepción de Munizaga (1974). Sin embargo, durante estos últimos años se ha complementado el conocimiento de este Complejo Cultural, con el estudio de sus restos bioantropológicos (Meller et al. 2009; Pacheco 2010; Pacheco y Retamal 2010; Retamal y Manríquez 2006; Retamal y Rivera 2005; Retamal et al. 2006, 2009; Urzúa et al. 2007). El presente trabajo continúa estas investigaciones, con el fin de aportar mayores datos a la discusión sobre este Complejo Cultural, desarrollando un estudio sistemático de la estimación del sexo, de la edad y de la longevidad de los individuos adultos de la colección cementerio Pica 8.

\section{$\checkmark$ Antecedentes}

Tradicionalmente, el Complejo Pica Tarapacá se ha interpretado como espacio de una sociedad con algún nivel de jerarquización y división social del trabajo (Núñez 1984), como un nodo de relaciones comerciales enfocado al tráfico caravanero entre costa y puna y como un centro multiétnico de intercambio (Zlatar 1984), que habría controlado localidades del litoral sur de Iquique y habría mantenido contactos con poblaciones altiplánicas, siguiendo el modelo de complementariedad de los recursos (Schiappacasse et al. 1989).

1 Departamento de Antropología, Universidad de Chile. Av. Ignacio Carrera Pinto 1045. Santiago, CHILE. Email: roretama@uchile.cl 
Respecto a su origen, Zlatar (1984) sugirió una proveniencia altiplánica, derivada de un grupo mayor del cual se desprendió para la formación de colonias o mitimaes, con el objetivo de controlar localidades como Bajo Molle, Los Verdes, Chucumata, Playa Brava y Patache-Cáñamo, entre otras (Sanhueza y Olmos 1985). Sin embargo, otras evidencias no descartan un origen derivado del contacto entre antiguas tradiciones costeras y grupos del interior (fase Cáñamo Montículo, 860 años AC; Núñez y Moragas 1983). Quienes plantean la existencia de un período Medio, sugieren que los individuos de este Complejo habrían convivido con población Tiawanaku asentada en las proximidades, siguiendo el modelo de Movilidad Giratoria (Núñez y Dillehay 1978).

Gran parte de estas interpretaciones se han basado en una concepción que vincula al Norte Grande durante el Intermedio Tardío con los procesos de los Andes Centrales, especialmente del área Circun-Titicaca, caracterizado por sociedades definidas como señoríos o sociedades de rango, que perseguían la autosuficiencia y la sustentabilidad social y económica, soportada bajo complejas estructuras sociales y refinados mecanismos de eco-complementariedad e interacción étnica (Murra 1972 y 1978; Núñez y Dillehay 1995). Esta concepción, basada principalmente en estudios etnohistóricos andinos, especialmente de Murra (1972, 1978 y 1983) se ha convertido en una suerte de paradigma de las sociedades preincaicas y ha impulsado enormemente el estudio de la prehistoria andina, privilegiando sus aspectos económicos y ecológicos (Albarracín-Jordán 1996; Llagostera 1995) y el ejercicio interdisciplinario para su interpretación a base de hallazgos etnohistóricos.

Sin embargo, algunos autores han observado que, en la actualidad, esta concepción ha estatizado la interpretación arqueológica a partir del estudio de la cultura material (Llagostera 1976; Uribe 1999-2000, 2006), utilizando los restos arqueológicos más bien para fortalecer ese modelo, sin una crítica sobre sus interpretaciones y obviando los aportes de la arqueología interpretativa, el estudio de la complejización de los sistemas sociales y la dinámica del poder (Earle 1991; Gutiérrez 1990; Miller y Tilley 1984).

\footnotetext{
2 Área de Arqueología, Universidad Bolivariana. Bandera 620. Santiago, CHILE: aryel.pacheco@gmail.com

3 Departamento de Antropología, Universidad de Chile. Av. Ignacio Carrera Pinto 1045. Santiago, CHILE: roretama@uchile.cl
}

Frente a esto, ha surgido una nueva línea de investigación que busca fortalecer la comprensión de las materialidades arqueológicas y su relación con los sistemas sociales de los Andes Centro-Sur durante el Intermedio Tardío (Albarracín-Jordán 1996; Gallardo et al. 1995; Lumbreras 1974; Nielsen 1995; Uribe y Adán 2000), compartiendo preceptos básicos de la arqueología post-procesual en relación al carácter multi-significativo de la cultura material y al rol interpretativo de la arqueología (Shanks y Hodder 1995).

Desde esta perspectiva, Uribe y Adán (2000, 2005) han planteado que la sociedad Pica Tarapacá buscaría la complementariedad de recursos como mantención de una tensión social, ayudando a ocultar ideológicamente sus propias contradicciones y desigualdades. A través de un estudio sistemático de este Complejo Cultural, Uribe (2006) ha descrito con mayor profundidad sus características, indagando en su complejidad y organización, más allá de los aspectos económicos y demográficos estudiados previamente (Núñez y Dillehay 1995 [1978]). Según Uribe (2006), reducir a esta sociedad bajo el término de señorío, ocultaría la enorme heterogeneidad de las diferentes comunidades que los constituyen, compuestas al menos por tres tradiciones diferentes que interactúan entre sí: una de raigambre en el Formativo Tardío, jerarquizada, centralizadora y exclusiva, propia de Tarapacá; otra, más cercana a los desarrollos septentrionales de los Valles Occidentales y el altiplano en la sierra, representada en un nivel más doméstico de organización del espacio; $y$, finalmente, una tradición costera enraizada desde finales del Arcaico e inicios del Formativo, autónomas en lo social, aunque articuladas económicamente con los valles (Núñez y Moragas 1983).

Las diferentes expresiones materiales caracterizarían la heterogeneidad de este Complejo Cultural, de acuerdo a estos tres referentes culturales (Uribe 2006). La arquitectura y la economía darían cuenta de la segmentación del sistema tradicional heredado del Formativo, de acuerdo a la dinámica histórica de acceso a los recursos y la naturaleza del paisaje. La alfarería denotaría la existencia de articulaciones entre distintas entidades que conforman el Complejo Pica Tarapacá, en tanto que los textiles, con características atribuibles al universo textil ariqueño, reafirmarían su inclusión dentro de la tradición de los Valles Occidentales, aunque con elementos propios (Uribe 2006). Paralelamente, los grupos costeros poseerían 
autonomía social, aunque mantendrían relaciones con grupos de quebradas, valles y oasis, trenzados mediante alianzas políticas, lo que se expresaría en contextos funerarios diferenciales (Moragas 1995; Núñez 1984).

Las características biológicas, observadas a partir del sitio Pica 8, también darían cuenta de una gran diversidad interna. Se observaría algún grado de especialización en el consumo de carbohidratos cariogénicos (Meller et al. 2009; Urzúa et al. 2007), división sexual del trabajo, diferencias sexuales o etarias en los peinados y deformaciones craneales, violencia doméstica, principalmente hacia el sexo femenino (Retamal et al. 2006), asociación entre la morfología facial y el grado de complejidad de su ajuar funerario (Retamal 2005), gran variedad de tipos de deformación craneal (Retamal y Manríquez 2006) y prácticamente ausencia de violencia intergrupal, a pesar de su manifestación a nivel simbólico (Pacheco y Retamal 2012).

Si bien estos avances en torno a la colección cementerio Pica 8 del norte de Chile han complementado el conocimiento sobre el Complejo Cultural Pica Tarapacá a nivel bioantropológico, hasta el momento no se ha realizado un estudio sistemático sobre su composición etaria y sexual, por lo que los estudios que se han realizado anteriormente presentan diferentes tamaños muestrales, lo que dificulta el entendimiento e interpretación de las características biológicas presentes en estos individuos. El conocimiento de la estructura sexual y etaria en una colección esqueletal es fundamental en la reconstrucción del perfil biológico de las poblaciones del pasado. Mediante este estudio, es posible conocer si la frecuencia etaria y sexual puede ajustarse a modelos poblacionales conocidos, o si denota problemas en la representación, explicados por la conservación o la recuperación preferencial de un sexo en particular (Hoppa y Vaupel 2002). Además, es uno de los primeros pasos para la reconstrucción de la estructura demográfica de estas poblaciones, si bien no se puede reconstruir directamente la estructura poblacional sobre la base de las estimaciones de edad mediante los métodos tradicionales (Hoppa y Vaupel 2002).

Junto con lo anterior, se prestó especial atención a los rangos etarios que representarían a los adultos mayores, con el fin de indagar sobre la presencia y frecuencia de los individuos más ancianos, como un primer acercamiento para entender la dinámica del envejecimiento dentro de este Complejo Cultural, con el propósito de enriquecer su interpretación desde una dimensión interna de su constitución cultural. Para Appleby (2010: 145), el proceso de envejecimiento es una parte fundamental de la vida de todo individuo y el reconocimiento social del envejecimiento es una parte importante de toda sociedad. Según esta autora, habrían tres implicaciones a la falta del estudio arqueológico del envejecimiento: primero, una falta de comprensión en la relación entre la vejez y la identidad de las sociedades prehistóricas. Segundo, una falla en entender la relación entre los cambios sociales en el modo de comprender la vejez y los cambios sociales en sí. Y tercero, no considerar el proceso del envejecimiento reforzaría una idea central dentro del pensamiento arqueológico, que es considerar al cuerpo como una entidad estable que no cambia en el curso de la adultez.

Para Appleby (2011: 146), el cambio en el pensamiento de los procesos asociados al envejecimiento sería vital en el proyecto de entender las identidades del pasado, ya que conseguiría conocer los procesos de desarrollo y cambio social. Al no explorar las acciones, intenciones y significados asociados a los miembros más ancianos de las comunidades prehistóricas, se arriesgaría la comprensión de estas comunidades. Esto es particularmente pertinente cuando se trata de entender lo mecanismos sociales que producen las diferencias sociales institucionalizadas.

En este sentido, consideramos que la heterogeneidad cultural de los grupos que conforman el Complejo Pica Tarapacá también podría manifestarse en sus características sexuales y etarias, como se ha observado en estudios anteriores (Retamal 2005; Retamal et al. 2006), pero que necesitan ser reforzados con estudios sistemáticos que consideren la mayor muestra posible. Para indagar más en profundidad respecto a las características físicas de los individuos representados en sus restos mortales, es necesario sistematizar la estimación del perfil biológico en lo referente a lo etario y sexual.

\section{$*$ Material}

El sitio Pica 8 se ubica a los pies de la precordillera de los Andes (1350 m.s.n.m.), aproximadamente a $30 \mathrm{~km}$ al este de la localidad de Matilla y al oeste del 


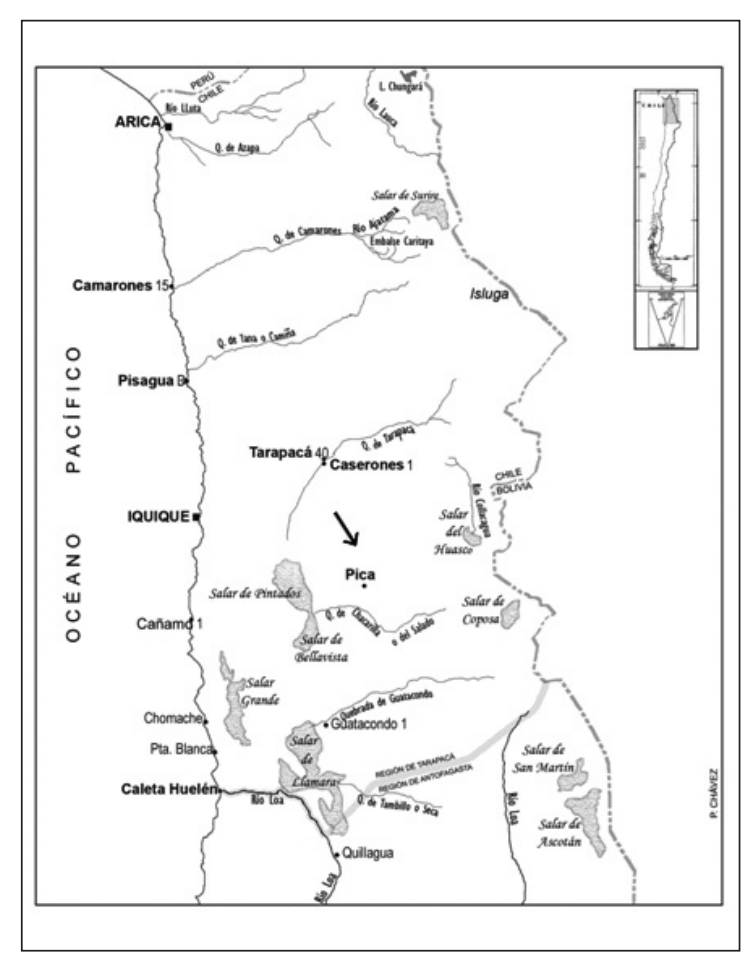

Figura 1. Ubicación geográfica del sitio Pica 8.

oasis de San Andrés de Pica (20 30'47.53" S; 69 21'33.15" W) (Figuras 1 y 2). El sitio fue excavado por Lautaro Núñez en una fecha anterior a 1964 (Gordon 1964), obteniendo 254 cuerpos enfardados y ofrendas asociadas (Núñez 1984: 248). Esta colección se encuentra actualmente depositada en el Departamento de Antropología de la Universidad de Chile y cuenta con 150 individuos en distinto grado de completitud y estado de conservación, tales como restos enfardados, momificados y esqueletizados.

Del material esqueletizado, se seleccionaron individuos adultos, considerando como su indicador la fusión de la articulación esfeno-occipital del cráneo, la fusión de los segundos núcleos de osificación del coxal y la fusión de los huesos largos (Schaefer et al. 2009). De ellos, se seleccionaron aquellos que presentaran al menos el cráneo o un coxal en buen estado de conservación. También se incluyeron en la muestra casos en donde solo se encontraron cráneos o coxales adultos. Mediante este procedimiento, la muestra del presente estudio se constituyó por 82 individuos (Tabla 1).

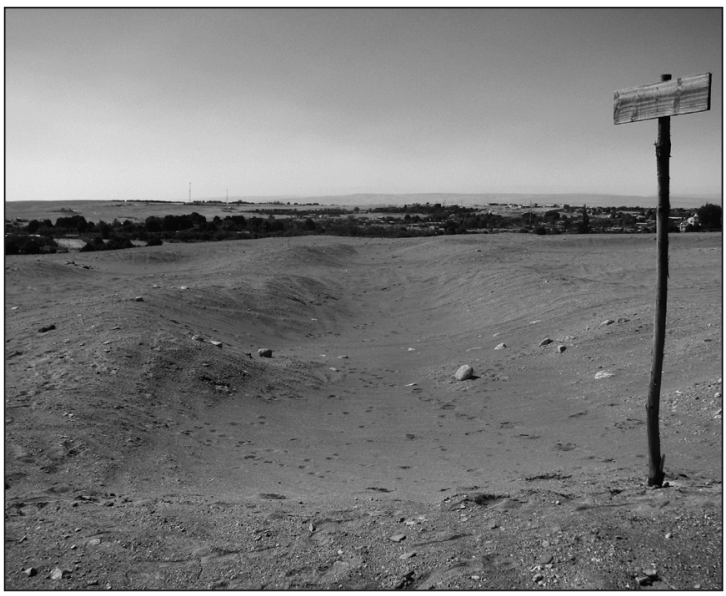

Figura 2. Vista general del sitio Pica 8 . Al fondo se observa la localidad de Matilla.

\section{* Métodos}

\section{Estimación del sexo}

Para la estimación del sexo, los cráneos y ambos coxales fueron separados en contenedores, se aleatorizaron y fueron analizados solo por un investigador (R.R.). Los rasgos utilizados fueron el arco ventral del pubis (A.V.), la concavidad subpúbica (C.S.), la rama ascendente del isquion (R.A.I.), la escotadura ciática mayor (E.C.M.) y el surco preauricular (S.P.), siguiendo las indicaciones de Buikstra et al. (1994). Se analizó la concordancia en la estimación entre el lado derecho e izquierdo de cada individuo y la concordancia entre pares de rasgos, utilizando la prueba de concordancia Kappa de Cohen (Cohen 1960), rechazando la hipótesis nula de que las concordancias se deban al azar, con un valor de probabilidad menor a 0,05. Para interpretar los valores de Kappa, se utilizó la escala de valores de Landis y Koch (1977). Con el fin de comparar las variables entre so, S.P. y E.C.M. fueron transformadas en variables dicotómicas, considerando como "femenino" valores bajo 3 para la E.C.M. y bajo 2 para S.P. Como "masculino" se asumió valores sobre 3 para la E.C.M. y para el S.P.

En cráneos, el dimorfismo sexual se estimó utilizando el método de Ferembach et al. (1980) y Buikstra et al. (1994). Valores entre -2 y - 0.2 fueron considerados femeninos, entre -0.19 y 0.19 fueron considerados indeterminados 


\begin{tabular}{|l|c|c|}
\hline \multicolumn{1}{|c|}{ Tipo } & $\mathbf{n}$ & $\%$ \\
\hline Ambos coxales y cráneo & 22 & 26,83 \\
\hline Un coxal y cráneo & 2 & 2,44 \\
\hline Ambos coxales & 11 & 13,41 \\
\hline Un coxal & 14 & 17,07 \\
\hline Cráneos & 33 & 40,24 \\
\hline TOTAL & 82 & $\mathbf{1 0 0 , 0 0}$ \\
\hline
\end{tabular}

Tabla 1. Frecuencia de elementos óseos en la muestra analizada.

\begin{tabular}{|c|c|c|}
\hline Rango etario & Brooks y Suchey (1990) & Lovejoy (1985) \\
\hline $20-29$ & I y II & 1 y 2 \\
\hline $30-39$ & III & 3 y 4 \\
\hline $40-49$ & IV & 5 y 6 \\
\hline $50-59$ & V & 7 \\
\hline $60+$ & VI & 8 \\
\hline
\end{tabular}

Tabla 2. Esquema de asociación entre cada fase de estimación a partir de los métodos utilizados con las categorías etarias decenales.

y entre 0.2 y 2 fueron considerados masculinos (Ferembach et al. 1980). En individuos que presentaron el cráneo y al menos un coxal, se estimó el sexo en ambos segmentos y se revisó la variación del dimorfismo sexual del cráneo, considerando como primer criterio el sexo estimado en el coxal, ya que éste expresaría el dimorfismo sexual con mayor certeza y precisión que otros indicadores (Klepinger 2006). En aquellos individuos que presentaron solamente el cráneo, se comparó el índice de sexualización (Ascadi y Nemeskeri 1970; Ferembach et al. 1980) con aquellos que presentaron coxal y cráneo para cada sexo. Para esto, se utilizó la prueba T de Student, con el fin de conocer si la distribución del índice de sexualización para cada sexo estimado mediante el cráneo presentaba diferencias significativas respecto a los individuos que presentaron cráneo y coxal. Se rechazó la hipótesis nula con un valor de probabilidad inferior a 0,05 .

\section{Estimación de la edad}

La edad de cada individuo fue estimada a partir de la sínfisis púbica y de la superficie auricular del ilion, si- guiendo los métodos de Brooks y Suchey (1990) y Lovejoy y colaboradores (1985), respectivamente. Ambas estimaciones se ajustaron para estimar rangos etarios de diez años, desde los veinte hasta los sesenta a más años. De este modo, el rango 20-29 años se asocia a las fases I y II de Brooks y Suchey (op. cit.) y 1 y 2 de Lovejoy (op. cit.), en tanto que los rangos decenales subsiguientes se asocian a las fases III, IV, V y VI de Brooks y Suchey (op. cit.) y a las fases 3-4, 5-6, 7 y 8 de Lovejoy (op. cit.) (Tabla 2).

Se evaluó la concordancia de cada rasgo entre coxales derechos e izquierdos y entre ambos métodos en cada coxal, utilizando la prueba Kappa de Cohen (Cohen 1960), rechazando la hipótesis nula de que las concordancias se deban al azar, con un valor de probabilidad menor a 0,05. Para interpretar la concordancia obtenida en la prueba Kappa de Cohen, se consideró la escala de interpretación propuesta por Landis y Koch (1977). En caso de que estos métodos presentaran diferencias en el rango etario estimado, se privilegió la estimación de Brooks y Suchey (op. cit.), ya que trabajos previos han reportado que el método de Lovejoy (op. cit.) subestimaría la edad, especialmente en los rangos etarios más avanzados (Murray y Murray 1991, Osbourne et al. 2004)

\section{Longevidad en Pica 8}

Una vez estimada la edad de los individuos, se clasificó a cada uno dentro de tres categorías etarias amplias: adulto joven (18 a 35 años), adulto medio ( 35 a 50 años) y adulto mayor (mayor de 50 años), siguiendo a Buikstra et al. (1994). Se describió y comparó la frecuencia entre cada rango etario y su asociación con el sexo. Para esto, se aplicó la prueba de Jiz para probar la asociación entre el sexo y cada rango etario, rechazando la hipótesis de no asociación entre el sexo y las categorías etarias con un valor de probabilidad menor a 0,05 .

\section{$*$ Resultados}

\section{Estimación del sexo}

Los resultados de las evaluaciones para cada rasgo entre coxales derechos e izquierdos se resumen en la Tabla 


\begin{tabular}{|c|c|c|c|c|c|}
\hline Variable & $\mathbf{n}$ (pares) & \% Concordancia & Kappa & Z & p-valor \\
\hline A.V. & 26 & 100 & 1 & 5,1 & $\ll 0,05$ \\
\hline C.S. & 26 & 100 & 1 & 5,1 & $\ll 0,05$ \\
\hline R.A.I. & 30 & 93,3 & 0,86 & 5,3 & $\ll 0,05$ \\
\hline S.P. & 32 & 90,63 & 0,84 & 6,59 & $\ll 0,05$ \\
\hline S.P. (o/1) & 32 & 100 & 1 & 6,48 & $\ll 0,05$ \\
\hline E.C.M. & 11 & 75,8 & 0,67 & 7,01 & $\ll 0,05$ \\
\hline E.C.M. (o/1) & 11 & 100 & 1 & 7,34 & $\ll 0,05$ \\
\hline
\end{tabular}

Tabla 3. Resultados de las prueba Kappa de Cohen en la estimación del sexo entre coxales derecho e izquierdo en cada individuo. A.V.: Arco ventral; S.P.: Surco preauricular; C.S.: Concavidad subpúbica; R.A.I.: Rama ascendente del isquion; E.C.M.: Escotadura ciática mayor. Los valores (o/1) indican que el rasgo fue transformado en una variable dicotómica.

\begin{tabular}{|c|c|c|c|c|}
\hline Par-Variable & \% Concordancia & Kappa & $\mathbf{Z}$ & p-valor \\
\hline A.V.-C.S. & 100 & 1 & 5,1 & $\ll 0,05$ \\
\hline A.V.-S.P. & 100 & 1 & 5,1 & $\ll 0,05$ \\
\hline A.V.-R.A.I. & 92.31 & 0,86 & 4,91 & $\ll 0,05$ \\
\hline A.V.-E.C.M. & 96.2 & 0,93 & 5 & $\ll 0,05$ \\
\hline C.S.-R.A.I. & 91.5 & 0,84 & 6,58 & $\ll 0,05$ \\
\hline C.S.-E.C.M. & 95.1 & 0,91 & 6,25 & $\ll 0,05$ \\
\hline C.S.-S.P. & 97.6 & 0,95 & 6,09 & $\ll 0,05$ \\
\hline R.A.I.-E.C.M. & 85 & 0,73 & 5,73 & $\ll 0,05$ \\
\hline R.A.I.-S.P. & 87.5 & 0,76 & 5,64 & $\ll 0,05$ \\
\hline S.P.-E.C.M. & 92.9 & 0,86 & 6,03 & $\ll 0,05$ \\
\hline
\end{tabular}

Tabla 4. Resultados de la prueba Kappa de Cohen para la concordancia en la estimación entre pares de rasgos. A.V.: Arco ventral; S.P.: Surco preauricular; C.S.: Concavidad subpúbica; R.A.I.: Rama ascendente del isquion; E.C.M.: Escotadura ciática mayor.

3. Todas las variables presentaron un valor Kappa muy bueno, a excepción de la escotadura ciática (bueno/sustancial). En todas las variables se rechazó la hipótesis de nulidad de que las concordancias se deban al azar. Cuando la escotadura ciática mayor y el surco preauricular se transformaron en variables dicotómicas $(\mathrm{o} / \mathrm{l})$, lograron obtener un $100 \%$ de concordancia.

El grado de concordancia entre pares de rasgos se resume en la Tabla 4. Se observa que todos presentan valores de Kappa muy buenos y significación estadística, a excepción del par R.A.I-E.C.M., que presentó un valor Kappa de 0.73 (bueno/sustancial). Con esta información, se logró estimar el sexo en 49 esqueletos ( 27 femeninos y 22 masculinos).

\section{Estimación del sexo utilizando el cráneo}

La tabla 5 muestra los resultados del índice de sexualización obtenido en el cráneo, para cada sexo estimado en su coxal. Para los individuos estimados femeninos por el coxal, el promedio del índice de sexualización fue de -1.07 y una desviación estándar de 0.52. Para el sexo masculino estimado en el coxal, el promedio del índice de sexualización fue de 1.21 y una desviación estándar de 0.53. Uno de los esqueletos (Sector F, tumba 4, inventario núm. Bo042), estimado femenino a partir de sus coxales, obtuvo un índice de sexualización de o.18 (indeterminado).

De los 33 cráneos que no presentaron coxal, 20 fueron estimados femeninos, con un índice de sexualización 


\begin{tabular}{|c|c|c|c|c|c|}
\hline Sexo & $\mathbf{n}$ & Media & Desv. Est. & Mínimo & Máximo \\
\hline Femenino & 17 & $-1,07$ & 0,53 & -2 & 0,18 \\
\hline Masculino & 7 & 1,21 & 0,53 & 0,68 & 2 \\
\hline
\end{tabular}

Tabla 5. Resultados del índice de sexualización en cráneos de individuos con sexo estimado por coxal.

\begin{tabular}{|c|c|c|c|c|c|}
\hline Cráneos & $\mathbf{n}$ & Media & Des. Est. & Mín & Máx \\
\hline Femeninos & 20 & $-1,069$ & 0,437 & $-1,955$ & $-0,046$ \\
\hline Masculinos & 13 & 1,1 & 0,389 & 0,591 & 1,727 \\
\hline
\end{tabular}

Tabla 6. Resultados del índice de sexualización en cráneos que no presentaron coxales asociados.

\begin{tabular}{|c|c|c|}
\hline Sexo & Frecuencia & Porcentaje \\
\hline Femenino & 47 & 57,3 \\
\hline Masculino & 35 & 42,7 \\
\hline Total & 82 & 100 \\
\hline
\end{tabular}

Tabla 7. Distribución del sexo estimado en la muestra.

\begin{tabular}{|c|c|c|c|c|c|}
\hline Tipo & $\mathbf{n}$ (pares) & \% acuerdo & Kappa & $\mathbf{Z}$ & p-valor \\
\hline Superficie auricular & 33 & $75,76 \%$ & 0,66 & 6,6 & $\ll 0,05$ \\
\hline Sínfisis púbica & 24 & $87,50 \%$ & 0,83 & 7,2 & $\ll 0,05$ \\
\hline
\end{tabular}

Tabla 8. Resultados de las pruebas de concordancia Kappa de Cohen en la estimación de la edad de muerte entre coxales derechos e izquierdos.

\begin{tabular}{|c|c|c|c|c|}
\hline $\mathbf{n}$ & \% Acuerdo & Kappa & $\mathbf{Z}$ & p-valor \\
\hline 25 & $24,00 \%$ & 0,15 & 2,81 & 0,0025 \\
\hline
\end{tabular}

Tabla 9. Resultados de la prueba de concordancia Kappa de Cohen en la estimación de la edad de muerte entre Brooks y Suchey (1990) y Lovejoy (1985).

\begin{tabular}{|c|c|c|c|c|c|c|c|}
\hline \multicolumn{2}{|c|}{} & \multicolumn{5}{|c|}{ Sínfisis púbica } & \\
\cline { 2 - 8 } \multicolumn{2}{|c|}{} & $\mathbf{2 0 - 2 9}$ & $30-39$ & $40-49$ & $50-59$ & $60+$ & Total \\
\hline \multirow{4}{*}{$\begin{array}{c}\text { Superficie } \\
\text { auricular }\end{array}$} & $\mathbf{2 0 - 2 9}$ & 5 & 0 & 0 & 0 & 0 & 5 \\
\cline { 2 - 8 } & $30-39$ & 0 & 1 & 11 & 3 & 3 & 18 \\
\cline { 2 - 8 } & $40-49$ & 0 & 0 & 0 & 0 & 2 & 2 \\
\hline & Total & 5 & 1 & 11 & 3 & 5 & 25 \\
\hline
\end{tabular}

Tabla 10. Estimación de la edad de muerte mediante los métodos de Brooks y Suchey (1990) y Lovejoy (1985) en coxales que presentaron ambos rasgos.

promedio de -1.06 y una desviación estándar de 0.43. Los 13 cráneos restantes se estimaron masculinos, con un promedio de 1.1 y una desviación estándar de 0.39 (Tabla 6). Ninguno de ellos se halló en la zona de indeterminación y no hubo diferencias estadísticas en el índice de sexualización con los individuos que presentaron cráneo y coxal (femeninos, $\mathrm{p}$-valor $=0.998$; masculinos, $\mathrm{p}$-valor $=0.601$ ). Finalmente, el total de la muestra pudo ser sexado mediante los métodos empleados en este estudio (Tabla 7), observándose un mayor porcentaje de individuos femeninos dentro de la muestra estudiada.

\section{Estimación de la edad}

La Tabla 8 sintetiza los resultados obtenidos de la prueba de concordancia Kappa de Cohen en la estimación de cada método entre el lado derecho e izquierdo. La sínfisis púbica presentó un valor Kappa muy bueno, en tanto que superficie auricular presentó un valor Kappa bueno. En ambos se rechazó la hipótesis de nulidad.

La Tabla 9 muestra la comparación de la concordancia entre ambos rasgos $(n=25)$. Se observa un bajo porcentaje de concordancia y un valor de Kappa pobre. Sin embargo, no se rechazó la hipótesis de nulidad de que las coincidencias entre ambos métodos se deban al azar $(\mathrm{p}=0.0025)$. La Tabla 10 muestra las estimaciones de cada uno de los métodos agrupado por rangos decenales. Para los rangos etarios 20-29 y 30-39, ambos métodos coincidieron en su estimación, sin embargo, sobre el rango 30-39 en adelante, no existen coincidencias entre ellos. El método de la superficie auricular pareciera estancarse en la estimación del rango 30-39, mientras que para los mismos casos, la sínfisis púbica estima edades hasta los 60 y más años. 


\begin{tabular}{|c|c|c|c|c|}
\hline Sexo & $\mathbf{2 0 - 2 9}$ & $30-39$ & $40-49$ & Total \\
\hline Femenino & $3(5,17 \%)$ & $9(15,52 \%)$ & $22(37,93 \%)$ & $34(58,62 \%)$ \\
\hline Masculino & $2(3,45 \%)$ & $9(15,52 \%)$ & $13(22,41 \%)$ & $24(41,38 \%)$ \\
\hline Total & $5(8,62 \%)$ & $\mathbf{1 8}(31,03 \%)$ & $35(60,34 \%)$ & 58 \\
\hline
\end{tabular}

Tabla 11. Estimación de sexo y edad, siguiendo las estimaciones de edad de Lovejoy (1985). Frecuencias relativas se muestran entre paréntesis.

\begin{tabular}{|c|c|c|c|c|c|c|}
\hline Sexo & $\mathbf{2 0 - 2 9}$ & $\mathbf{3 0 - 3 9}$ & $\mathbf{4 0 - 4 9}$ & $\mathbf{5 0 - 5 9}$ & $\mathbf{6 0 +}$ & Total \\
\hline Femenino & $4(8,6 \%)$ & $1(2,1 \%)$ & $5(10,8 \%)$ & $6(13 \%)$ & $9(19,6 \%)$ & $25(54,3 \%)$ \\
\hline Masculino & $5(10,8 \%)$ & 0 & $12(26,1 \%)$ & $4(8,6 \%)$ & 0 & $21(45,7 \%)$ \\
\hline Total & $9(19,6 \%)$ & $1(2,1 \%)$ & $\mathbf{1 7}(36,9 \%)$ & $10(21,7 \%)$ & $9(19,6 \%)$ & 46 \\
\hline
\end{tabular}

Tabla 12. Estimación del sexo y la edad de muerte siguiendo las estimaciones de edad de Brooks y Suchey (1990). Frecuencias relativas se muestran entre paréntesis.

\begin{tabular}{|c|c|c|c|c|}
\hline Sexo & Adulto Joven & Adulto Medio & Adulto Mayor & Total \\
\hline Femeninos & $5(11 \%)$ & $5(11 \%)$ & $15(32 \%)$ & $\mathbf{2 5}(54 \%)$ \\
\hline Masculinos & $5(11 \%)$ & $12(26 \%)$ & $4(9 \%)$ & $\mathbf{2 1}(46 \%)$ \\
\hline Total & $\mathbf{1 0}(\mathbf{2 2} \%)$ & $\mathbf{1 7}(37 \%)$ & $\mathbf{1 9}(41 \%)$ & 46 \\
\hline
\end{tabular}

Tabla 13. Sexo y edad al morir en categorías etarias amplias, siguiendo las estimaciones de edad de Brooks y Suchey (1990). Frecuencias relativas se muestran entre paréntesis.

La Tabla 11 presenta el perfil etario y sexual de la muestra, siguiendo las estimaciones de Lovejoy (1985). Esta tabla muestra que existe un incremento en la cantidad de individuos a medida que aumenta la edad estimada. Sobre el 60\% de la muestra presenta edades de muerte entre 40-49 años. Por otra parte, la relación entre ambos sexos muestra que el sexo femenino presenta mayor proporción que el sexo masculino.

La Tabla 12 muestra las estimaciones del sexo y la edad mediante las estimaciones de edad de Brooks y Suchey (1990). Se observa una baja frecuencia de casos durante las primeras décadas de adultez, una mayor proporción entre los 40 y 49 años y un descenso en los rangos etarios posteriores. En el sexo femenino, las frecuencias son menores que el sexo masculino, a excepción del rango sobre
60 años. El sexo masculino presenta una baja frecuencia entre 20 a 39 años, se incrementa notablemente entre los 30 a 49 años y luego decrece entre los 50 a 59 años, sin que se observen casos sobre los 60 años.

Dado que trabajos previos han constatado que el método de Lovejoy (1985) subestimaría la edad, especialmente en los rangos etarios más avanzados (Murray y Murray 1991; Osbourne et al. 2004), se siguió el estudio con las estimaciones de Brooks y Suchey (1990). Se construyeron categorías etarias amplias, indicadas en la Tabla 13. La proporción de adultos jóvenes (20-35 años) es de un $22 \%$, de adultos medios (35-49 años) un $37 \%$ y de adultos mayores (sobre 50 años) de un $41 \%$. El test de Ji2 muestra que existe asociación entre el sexo y las categorías etarias amplias de edad de muerte estimada en esta colección $(\mathrm{Ji2}(2$ g.l. $))=8.97$, p-valor $=0.011)$.

En síntesis, mediante las metodologías empleadas para la estimación del sexo, se pudo sexar a toda la muestra. Respecto a la edad, se prefirió el método de Brooks y Suchey (1990), en donde se pudieron estimar 46 individuos de acuerdo a las tres categorías etarias amplias. Se halló asociación entre el sexo y la edad de muerte dividida en rangos etarios amplios. El Anexo 1 muestra el sexo y la edad estimada por ambos métodos para cada individuo de la muestra estudiada.

\section{* Discusión}

\section{Estimación del sexo}

Los resultados de las estimaciones del sexo tuvieron muy buenos valores de acuerdo entre lados derecho e izquierdo, a excepción de escotadura ciática mayor, que alcanzó un valor levemente más bajo, sugiriendo una alta asimetría bilateral de estos indicadores. Según Klepinger (2006), los hitos del coxal pueden ser considerados el "Gold Standard" de la identificación del sexo en el ser humano, debido a las presiones selectivas que sufre la pelvis femenina para permitir el parto. Cuando se analizaron pares de estimadores, las pruebas Kappa de Cohen mostraron significación estadística en todos los pares analizados y muy buenos valores de acuerdo, a excepción de los pares rama ascendente del isquion-surco preauricular y rama ascendente del isquion-escotadura 
ciática mayor, que mostraron valores Kappa levemente más bajos. Lo anterior sugiere que existe una alta precisión y certeza de las estimaciones del sexo entre estos hitos anatómicos, lo que ha sido reportado anteriormente para otras colecciones (Phenice 1969, St. Hoyme e Iscan 1989, Sutherland y Suchey 1991). La escotadura ciática mayor y el surco preauricular presentaron los puntajes más bajos de concordancia, debido posiblemente a que estos métodos inducen a mayores errores en la estimación entre grados cercanos.

La estimación del sexo en cráneos mostró resultados muy similares a la estimación por coxales. En la zona de indeterminación $(-0,2-+0,2$, Ascadi y Nemeskeri 1970), se halló solamente un esqueleto femenino (Sector F, tumba 4, inventario núm. Boo42). Según White y Folkens (2005: 386), si bien el sexo femenino en el humano se caracteriza por ser morfológicamente más grácil y pequeño en comparación al masculino, la variación individual normal dentro de una población produce a menudo mujeres robustas y hombres gráciles, que convergen en el centro de la distribución, en donde el sexo es más difícil de estimar. Más precisamente, cuando el dimorfismo sexual se analiza a partir de variables métricas, cada sexo presentaría una función de distribución, generalmente gaussiana (normal), que se superponen entre ellas, en mayor o menor medida de acuerdo a su expresión del dimorfismo sexual (Chakraborty y Majumder 1982; Ipiña y Durand 2000, 2004; Josephson et al. 1996; Marini et al. 1999). Si bien el índice de sexualización releva el dimorfismo sexual utilizando un set de variables categóricas ordinales, estas variables describen rasgos morfológicos en donde subyace un componente de naturaleza continua. Para el caso de la colección Pica 8 , este índice muestra que pueden existir cráneos femeninos bastante robustos como para entrar en la zona de indeterminación de la estimación, lo que implica un hecho normal de la variación craneana. De todos modos, no se pueden descartar otros factores, tales como el envejecimiento (Meindl et al. 1985).

Una vez obtenidas las proporciones de ambos sexos, llamó la atención la disparidad en la proporción entre esqueletos femeninos y masculinos dentro de la muestra. Esta notoria tendencia hacia una mayor proporción de esqueletos femeninos podría estar reflejando problemas muestrales, posiblemente asociados a la recuperación de la colección o a su posterior manejo, lo que tiene que corroborarse en una muestra mayor a la utilizada en este estudio.

\section{Estimación de la edad de muerte}

Respecto a la estimación de la edad, se observó la presencia de coincidencias en las estimaciones solamente en los primeros dos rangos etarios decenales (20-29 y 30-39). Sin embargo, desde los 40 años en adelante, las estimaciones fueron dispares, mostrando que el método de Lovejoy (1985) solo estimó edades de muerte hasta los 40-49 años, lo que influye en la elevada proporción de individuos estimados en este rango etario (sobre el 60\%; Tabla 11). Resultados similares se han encontrado en trabajos que han utilizado muestras con edad documentada, mostrando que este método subestima la edad en los rangos etarios más avanzados (Murray y Murray 1991; Osbourne et al. 2004; Schmidt 1994). Por lo tanto, es posible que el método de Lovejoy et al. (1985) también subestime la edad al morir de los individuos adultos mayores de esta colección. Por su parte, el método de Brooks y Suchey (1990) estimó la edad al morir hasta los sesenta o más años, atribuyendo este rango al sexo femenino. Esto sugiere una mayor longevidad del sexo femenino sobre el masculino, aunque se requiere una muestra más amplia y una mayor cantidad de métodos para corroborar esta interpretación.

La distribución etaria de acuerdo a las categorías amplias de adultez (joven, medio, mayor), muestra una baja frecuencia de adultos jóvenes (22\%) y una alta frecuencia de adultos mayores (41\%). El test de Ji2 para conocer la asociación entre el sexo y la edad en categorías amplias, presentó significación estadística, sugiriendo diferencias en las edades de muerte entre individuos masculinos y femeninos a lo largo de los rangos etarios. Sin embargo, ha que tener presente que lo anterior también puede deberse a problemas muestrales, por lo que se necesitaría una muestra mayor y más métodos de estimación de la edad.

Es interesante la elevada frecuencia de adultos jóvenes y medios observado en el presente estudio, que alcanzan el $59 \%$ en las estimaciones basadas en el método de Brooks y Suchey (1990) y en el 100\% de las estimaciones basadas en Lovejoy (1985). Hallazgos similares se han observado en otras colecciones esqueletales de diferente 
proveniencia y temporalidad (Chamberlain 2006: 90) y difieren de las distribuciones etarias realizadas a partir de datos demográficos históricos o modelos demográficos aceptados internacionalmente. Algunos autores consideran que este fenómeno se debe a una preservación preferencial hacia los adultos jóvenes y medios en el registro arqueológico (Walker et al. 1988). Otros asumirían que es reflejo de una longevidad reducida de las poblaciones del pasado (Meindl y Russell 1998; Meindl et al. 1983); es decir, un envejecimiento más rápido que en tiempos modernos, lo que conllevaría a una reducida vitalidad de las poblaciones (Chamberlain 2006: 53), que implicaría una ausencia de adultos mayores y un número aumentado de individuos infantiles huérfanos (Crawford 1999; Howell 1982).

Sin embargo, se ha mostrado que esto corresponde a un sesgo sistemático en la estimación de la edad utilizando indicadores esqueletales, respecto a datos demográficos históricos o etnográficos, lo que se traduce en una sobrestimación de adultos jóvenes y medios y una subestimación de adultos mayores (Boddington 1987: 190; Weiss 1973: 59). La subestimación de los adultos mayores ha sido uno de los temas centrales de discusión dentro del estudio paleodemográfico (Hoppa y Vaupel 2002), como parte de los problemas de estimación de la edad de muerte basado en indicadores esqueletales. Si bien estos estimadores morfológicos presentan algún grado de correlación con la edad cronológica (Arking 1998), se encontrarían más bien asociados con el estatus fisiológico del individuo (Kemkes-Grottenhaler 2002: 49). Por esto, aunque el proceso de envejecimiento es universal, en la medida en que afecta a todas las poblaciones y a ambos sexos, presenta una notable heterogeneidad interpersonal debido a variaciones genéticas, del comportamiento y otras predisposiciones, además de la interacción entre cada individuo con su ambiente (Kemkes-Grottenhaler 2006: 49), lo que se manifiesta también en una notable variación morfológica intra-individual (Spirduso 1995).

Es importante recalcar que los problemas de estimación tienden a subestimar la edad cronológica de los adultos mayores, por lo que, si bien no se puede tener certeza de la edad cronológica, sí sugieren que al menos cada individuo presenta cierta edad estimada. Siguiendo esta idea, los individuos que presentaron fase VI (intervalo de confianza de 42 a 87 años) mediante Brooks y Suchey (1990), tendrían al menos unos 42 años, es decir desde el final de la adultez media. Ya que fueron solamente estimados individuos femeninos dentro de este rango etario, estos individuos se encontrarían al menos a comienzos del climaterio. Por lo tanto, en base a estos resultados, en el presente trabajo es posible sugerir la presencia de individuos adultos mayores dentro de la colección Pica 8. Si bien su número es menor dentro del grupo ( 9 casos, correspondiente al 11\% de la muestra total), es notoria su presencia dentro de los individuos que presentaron solamente sínfisis púbicas (cerca de un 20\%), por lo que podría esperarse encontrar más casos de acuerdo a la mayor presencia de este segmento anatómico.

Diversas fuentes etnográficas e históricas muestran que los adultos sobre sesenta años seguían siendo parte activa en diversas sociedades con características demográficas muy disimiles entre ellas (Blurton Jones et al. 1992; Chamberlain 2006: 59, 61, 66-67; Harlow y Laurence 2002: 118; Headland 1989; Harrison 1995; Hill y Hurtado 1995; Howell 1979; Janetta y Preston 1991; Sarkar 1951; Scott y Duncan 1998; Shahar 1993; Smith 1960; Tomasson 1977), por lo que no sería extraño hallar individuos adultos mayores y seniles dentro del Complejo Pica Tarapacá.

\section{Longevidad en el pasado}

Al encontrarse con la presencia de individuos de edad avanzada dentro de esta colección, aparecen interrogantes como: ¿Qué rol social y cultural habrían tenido los ancianos dentro del Complejo Pica Tarapacá? ¿De qué forma se establecieron, como indica Beauvoir (1970), las soluciones de compromiso entre la sociedad y sus ancianos? ¿Fueron integrados o desechados del quehacer social? Vinculado a lo anterior, ilos datos bioantropológicos y arqueológicos disponibles para esta colección permitirían responder a estas preguntas?

Riley (1984: 8) plantea que el envejecimiento debe ser entendido desde su variabilidad sociocultural, ya que, como La Fontaine afirma (1986: 2), las diferenciaciones sexuales y etarias no son solo "hechos de la vida" sino también "hechos de la cultura". Appleby (2011) sugiere examinar tres aspectos de la edad: la edad cronológica, la edad biológica y la edad social. Si bien el envejeci- 
miento se encuentra ligado a la cronología, no es determinado por ella, incluso en tiempos modernos. Hay que tener en cuenta que en el registro bioantropológico, la edad cronológica se estima a partir de la edad biológica, que corresponde más bien a un diagnóstico del estado de la degeneración esqueletal, fuertemente influido por diferencias poblacionales y estilos de vida individuales. El factor clave no es solo que la edad cronológica no puede ser calculada directamente a partir del esqueleto, sino que el envejecimiento es mucho más que la edad cronológica. Los factores genéticos y biológicos involucrados en el proceso del envejecimiento influyen en las construcciones sociales de la identidad del envejecimiento, como factores que median en la forma en que son expresados (Appleby 2011: 150). Las personas no solo son clasificadas por su edad cronológica, sino también por su apariencia física y comportamiento, por lo que individuos de edades relativamente diferentes pueden tener un aspecto y comportamiento similar $y$ ser tratados similarmente por el entorno. Al contrario, individuos de edad similar pueden tener comportamientos y apariencias distintas, por lo que la sociedad construye una imagen diferente de ellos. Por lo tanto, es importante apartarse del foco de la edad cronológica como indicador exclusivo de la vejez, hacia un entendimiento más complejo del fenómeno. En este sentido, la edad biológica y la edad social son particularmente útiles en interpretar los significados de la edad en el contexto arqueológico. Siguiendo esta metodología, Appleby (2011) considera que es posible rescatar datos sobre la identidad del sujeto y de su entorno a partir del contexto arqueológico, con el fin de conocer cómo la gente se entendía a ella misma y cómo las entendía el entorno social.

Usar el esqueleto y su contexto como fuente de varias facetas del proceso de envejecimiento es una reconstrucción que se realiza por diferentes vías. Esto permite la investigación de la relación entre diversos factores y las construcciones sociales del envejecimiento. Hay que tener presente que la edad biológica no actúa sola para definir la identidad social, sino que también intervienen otras características, tales como el género, el parentesco, el estatus social y la afiliación étnica y religiosa, entre otras (Appleby 2011). Por otra parte, consideramos importante congregar bajo esta perspectiva, diferentes fuentes de interpretación, tales como la etnografía, la etnohistoria, estudios evolutivos y comparaciones con otros estudios arqueológicos, entre otros, con el fin de aproximarse a este tema desde una perspectiva integral.

Evolutivamente, la presencia de adultos mayores en las sociedades humanas, especialmente mujeres, beneficiaría a sus parientes mediante un fenómeno denominado "selección de parentesco" (kin selection), en donde los cambios en las frecuencias génicas a través de las generaciones son debidas en parte, a interacciones entre individuos emparentados. Bajo selección natural, un gen que codifique un carácter que mejore la aptitud de los portadores, aumentaría su frecuencia en esta población o disminuiría si redujera su aptitud. Sin embargo, si un gen disminuye la aptitud del portador, pero beneficia la aptitud de sus parientes, aumentaría su frecuencia dentro de esa población. La mejora en la aptitud de los parientes compensaría la pérdida de aptitud sufrida por el individuo que expresa el carácter (Hamilton 1971, 1972, 1975; Maynard 1964).

Según esta hipótesis se ha sostenido que la menopausia y el prolongado tiempo de sobrevida post-reproductivo de las mujeres serían caracteres derivados en el humano, que le confiriere una serie de ventajas evolutivas, tanto al individuo como a su descendencia (Hawkes 2003; Williams 1957), protegiendo al individuo de los riesgos asociados al embarazo y la lactancia, fenómenos altamente perjudiciales para su sobrevida, y benefician a la progenie emparentada (hijos, sobrinos y nietos), mediante el cuidado y enseñanza, permitiendo aumentar la apropiación de recursos, la descendencia en la familia completa y la fertilidad poblacional (Hawkes 2003; Williams 1957). Esta hipótesis, conocida con el nombre de "hipótesis de la abuela" (Hawkes et al. 1998; Hawkes 2003; Williams 1957), se expresaría desde los orígenes de Homo sp. (Swisher et al. 1994; Walker y Leakey 1993) y ha mostrado una gran utilidad en la explicación de la estructura biológica humana, tales como la madurez tardía, el rápido crecimiento de los infantes, la aceleración del destete, el aumento de la fertilidad (Hawkes et al. 1998), la estructura y variabilidad social vinculada con los mecanismos de cooperación familiar y co-residencia entre mujeres y sus hijos (Hawkes 2003; Hawkes et al. 1997; Lahdenperä et al. 2004) y patrones de residencia en cazadores-recolectores modernos (Ember 1975, 1978; Murdok 1967). 
Desde un enfoque cultural, Beauvoir (1970) plantea una relación directa entre el grado de constricción ambiental o complejidad social y la integración del anciano en la sociedad. En sociedades que viven sometidas a presiones ambientales muy fuertes (extrema pobreza, climas duros, recursos insuficientes, etc.), los conocimientos tecnológicos, sociales y culturales mínimos disminuyen, así como la "utilidad" de los ancianos, apenas sus fuerzas comienzan a mermar. Al contrario, "[...] cuando la vida económica requiere un saber más rico, cuando la lucha contra la naturaleza es menos áspera [...], la magia y la religión se desarrollan $[. .$.$] y la función del anciano se hace entonces$ más compleja" (op. cit:: 74).

Por otra parte, Mead (1970) distingue tres tipos de cultura: "[...] posfigurativa, en la que los niños aprenden de sus mayores; cofigurativa, en la que tanto los niños como los adultos aprenden de sus pares, y prefigurativa, en la que los adultos también aprenden de los niños" (Mead 1970: 35). Para la autora, las sociedades primitivas y los pequeños reductos religiosos e ideológicos son principalmente posfigurativos (Mead 1970: 35), en donde la autoridad se extrae del pasado, el cambio cultural es lento y la identidad de la sociedad es conocida de lleno por sus ancianos, quienes proporcionarían la pauta básica a las generaciones futuras.

Respecto a la evidencia etnohistórica en el mundo andino, hay escasa documentación. Guaman Poma de Ayala (1615) ubica a las mujeres de cincuenta a ochenta años dentro de la segunda categoría o calle del sexo femenino, encargadas de tejer ropa gruesa de comunidad, servir como porteras, dispenseras, camareras, cocineras, mayordomas de mujeres principales y servir a las doncellas de alta alcurnia, además de ser "[...] biejas honradas [...]" (op. cit.), diferenciándolas de las mujeres de entre 30 a 50 años, encargadas de tejer ropa delicada y delgada. Es interesante la diferencia en el oficio del tejido entre la mujer joven y la de mayor edad, aparentemente confiriendo más importancia a las primeras, debido posiblemente a una mayor destreza manual y agudeza visual.

Cobo (1956 [1893]), al hablar de los ancianos en América, comenta un trato inhumano hacia ellos por parte de sus hijos: "[...] como empiezan los desventurados padres a ir decaeciendo y declinando con la vejez [...] los sujetan a su obediencia y servicio y no solo se sirven dellos como viles esclavos, sino que les hacen tan cruel e inhumano tratamiento como si fueran perros o otro animal inferior [...]" (op. cit.: 26). Además, destaca que son quienes padecen mayores necesidades y miseria, debido no solo a la merma de la fuerza para el trabajo, sino también porque no hay “[...] entre estos bárbaros cosa más vil y desechada que los viejos". Las marcadas diferencias entre ambos autores respecto a este tema deberían examinarse en profundidad, con el fin de conocer qué aportes entrega cada una de estas visiones tan diferentes al conocimiento de la vejez y el envejecimiento en el pasado.

\section{$*$ Conclusiones}

En síntesis, el presente trabajo estudia la distribución sexual y etaria de la colección Pica 8, con el propósito de sistematizar esta información para futuros trabajos sobre esta colección. No obstante, el material analizado no corresponde a la totalidad de la colección original excavada por Lautaro Núñez (1984), desconociéndose hasta el momento la ubicación del 41\% restante (100 esqueletos), ni tampoco se dispone de la información obtenida durante la excavación de este sitio. Es deber de las instituciones patrimoniales y de la comunidad arqueológica velar por el acceso apropiado a las colecciones patrimoniales presentes en nuestro país, con el fin de otorgar una puesta en valor acorde a su importancia.

Respecto a la estimación del sexo, este estudio logró estimar el sexo de todos los individuos, con un bajo grado de desacuerdo entre los diferentes métodos. Respecto a la estimación de la edad, se observó que el método de Brooks y Suchey (1990) estimó individuos sobre 60 años en tanto que Lovejoy (1985) solo logró estimar hasta los 40-49 años. Lo anterior podría estar indicando una subestimación de la edad de muerte en base a este último método.

No obstante, la presencia de individuos sobre los 60 años de edad abre nuevas preguntas sobre el Complejo Cultural Pica Tarapacá. Queda como interrogante conocer si su presencia refleja la capacidad de este Complejo Cultural de poseer las condiciones económicas y tecnológicas suficientes como para integrar y aprovechar a los ancianos como parte de su heterogeneidad cultural, que mediante el apoyo recíproco, permitirían una sociedad 
con mayores sofisticaciones culturales y materiales, generadas a partir de la tradición.

Queda abierta la invitación para estudiar la vejez en el pasado y en general, las diferentes fases de la vida en el registro arqueológico, con el fin de conocer a las sociedades del pasado, no desde fuera de ellas, sino desde su constitución interna. Esta es una invitación a estudiar las colecciones esqueletales y materialidad asociada, enfocándose desde una visión geroantropológica (Cohen 1994) y dentro de un enfoque mayor que integre la antropología de las edades. Posiblemente, esto permita conocer más la diversidad social de las culturas arqueológicas y motive un estudio paleodemográfico más acabado y profundo de los restos biológicos y culturales del pasado de nuestro país.

Agradecimientos Este proyecto fue parcialmente financiado por el proyecto FONDECYT 1030923 (investigador responsable, Mauricio Uribe). Agradecemos al Departamento de Antropología de la Universidad de Chile por el acceso a la colección. También agradecemos a la antropóloga Dra. Paulina Osorio y a la profesora Victoria Castro por sus aportes en la discusión del fenómeno gerontológico. Finalmente, les debemos nuestra gratitud a Estefanía Vidal y Elizabeth Shaeffer por su apoyo en la corrección del manuscrito.

\section{$*$ ANEXO}

Listado de cada esqueleto con sexo y edad estimadas (Edad SF: Edad Sínfisis púbica, Edad A: Edad aurícula).

\begin{tabular}{|c|c|c|c|}
\hline $\begin{array}{c}\mathbf{N}^{\circ} \\
\text { INVENTARIO }\end{array}$ & SEXO & EDAD SF & EDAD A \\
\hline bo 423 & femenino & $20-29$ & $20-29$ \\
\hline bo 499 coxder2 & femenino & $20-29$ & $20-29$ \\
\hline bo 499 coxizl & femenino & $20-29$ & $20-29$ \\
\hline bo 453 & femenino & $20-29$ & . \\
\hline $\operatorname{sit} 58$ & femenino & $30-39$ & $30-39$ \\
\hline bo 419 & femenino & $40-49$ & $30-39$ \\
\hline bo 420 & femenino & $40-49$ & $30-39$ \\
\hline bo 434 & femenino & $40-49$ & $30-39$ \\
\hline bo 433 & femenino & $40-49$ & . \\
\hline bo 439 & femenino & $40-49$ & . \\
\hline bo 430 & femenino & $50-59$ & $30-39$ \\
\hline bo 462 & femenino & $50-59$ & $30-39$ \\
\hline bo 426 & femenino & $50-59$ & . \\
\hline bo 463 & femenino & $50-59$ & . \\
\hline bo 468 & femenino & $50-59$ & . \\
\hline bo 485 & femenino & $50-59$ & . \\
\hline bo 425 & femenino & $60+$ & $30-39$ \\
\hline bo 438 & femenino & $60+$ & $30-39$ \\
\hline bo 478 & femenino & $60+$ & $30-39$ \\
\hline bo449 & femenino & $60+$ & $40-49$ \\
\hline bo 484 & femenino & $60+$ & $40-49$ \\
\hline
\end{tabular}

\begin{tabular}{|c|c|c|c|}
\hline $\mathrm{N}^{\circ}$ INVENTARIO & SEXO & EDAD SF & EDAD A \\
\hline bo510 & femenino & . & $40-49$ \\
\hline bo511 & femenino & . & $40-49$ \\
\hline pi8 6 & femenino & . & $40-49$ \\
\hline $\mathrm{sd} \mathrm{t54}$ & femenino & . & $40-49$ \\
\hline bo 416 & femenino & . & . \\
\hline bo 435 & masculino & $20-29$ & $20-29$ \\
\hline bo $467 b$ & masculino & $20-29$ & $20-29$ \\
\hline bo 444 & masculino & $20-29$ & . \\
\hline $\begin{array}{c}\text { bo } 465 \text { coxder } 3 \\
\text { coxiz } 6\end{array}$ & masculino & $20-29$ & . \\
\hline bo 465 coxiz7 & masculino & $20-29$ & . \\
\hline bo 427 & masculino & $40-49$ & $30-39$ \\
\hline bo 431 & masculino & $40-49$ & $30-39$ \\
\hline bo 441 & masculino & $40-49$ & $30-39$ \\
\hline bo 457 & masculino & $40-49$ & $30-39$ \\
\hline bo 465 coxder 5 & masculino & $40-49$ & $30-39$ \\
\hline bo 467 & masculino & $40-49$ & $30-39$ \\
\hline bo493 & masculino & $40-49$ & $30-39$ \\
\hline bo5o8 & masculino & $40-49$ & $30-39$ \\
\hline bo415 & masculino & $40-49$ & . \\
\hline bo 440 & masculino & $40-49$ & . \\
\hline
\end{tabular}




\begin{tabular}{|c|c|c|c|}
\hline $\begin{array}{c}\mathbf{N}^{\circ} \\
\text { INVENTARIO }\end{array}$ & SEXO & EDAD SF & EDAD A \\
\hline bo421 & femenino & $60+$ & $\cdot$ \\
\hline bo428 & femenino & $60+$ & $\cdot$ \\
\hline bo430 & femenino & $60+$ & $\cdot$ \\
\hline bo499 coxiz4 & femenino & $60+$ & $\cdot$ \\
\hline 80 & femenino &. & $40-49$ \\
\hline bo410 & femenino &. & $40-49$ \\
\hline bo412 & femenino &. & $40-49$ \\
\hline bo413 & femenino &. & $40-49$ \\
\hline bo422 & femenino &. & $40-49$ \\
\hline bo424 & femenino &. & $40-49$ \\
\hline bo432 & femenino &. & $40-49$ \\
\hline bo442 & femenino &. & $40-49$ \\
\hline bo448 & femenino &. & $40-49$ \\
\hline bo470 & femenino &. & $40-49$ \\
\hline bo472 & femenino &. & $40-49$ \\
\hline bo473 & femenino &. & $40-49$ \\
\hline bo495 & femenino &. & $40-49$ \\
\hline bo505 & femenino &. & $40-49$ \\
\hline bo506 & femenino &. & $40-49$ \\
\hline bo507 & femenino &. & $40-49$ \\
\hline
\end{tabular}

\begin{tabular}{|c|c|c|c|}
\hline $\mathbf{N}^{\circ}$ INVENTARIO & SEXO & EDAD SF & EDAD A \\
\hline bo 476 & masculino & $40-49$ & . \\
\hline bo499 coxizz & masculino & $40-49$ & . \\
\hline $\begin{array}{c}\text { bo499 coxiz3; } \\
\text { coxder } 4\end{array}$ & masculino & $50-59$ & $30-39$ \\
\hline bo 465 coxiz5 & masculino & $50-59$ & . \\
\hline bo 469 & masculino & $50-59$ & . \\
\hline bo 499 coxder1 & masculino & $50-59$ & . \\
\hline bo 447 & masculino & . & $40-49$ \\
\hline bo 454 & masculino & . & $40-49$ \\
\hline bo 455 & masculino & . & $40-49$ \\
\hline bo46o & masculino & . & $40-49$ \\
\hline bo471 & masculino & . & $40-49$ \\
\hline bo 475 & masculino & . & $40-49$ \\
\hline bo 489 & masculino & . & $40-49$ \\
\hline bo 490 & masculino & . & $40-49$ \\
\hline bo 496 & masculino & . & $40-49$ \\
\hline bo504 & masculino & . & $40-49$ \\
\hline bo512 & masculino & . & $40-49$ \\
\hline $\mathrm{sd} \mathrm{t3o}$ & masculino & . & $40-49$ \\
\hline si t 66 & masculino & . & $40-49$ \\
\hline bo 469 & masculino & . & . \\
\hline
\end{tabular}

\section{* Referencias citadas}

ALBARRACÍN-JORDÁN, J., 1996. Tiwanaku. Arqueología regional y dinámica segmentaria. Editorial Plural, La Paz.

APPLEBY, J., 2010. Why We Need an Archaeology of Old Age, and a Suggested Approach. Norwegian Archaeological Review 43 (2): $145-168$.

ARKING, R., 1998. Biology of aging. Observations and principles. Sinauer Associates Inc, Sunderland.

ASCADI, G., y J. NEMESKERI, 1970. History of human lifespan and mortality. Akademiai Kiaido, Buddapest.

BEAUVOIR, S., 1970. La Vejez. Editorial Sudamericana, Buenos Aires.

BLURTON JONES, N. G., L. C. SMITH, J. F. O'CONNELL, K. HAWKES y C. KAMUZORA, 1992. Demography of the Hadza, an increasing and high density population of savanna foragers. American Journal of Physical Anthropology 89: 159-181.
BODDINGTON, A., 1987. From bones to population: the problem of numbers. En 1987 Death Decay and Reconstruction: Approaches to Archaeology and Forensic Science, A. Boddington, A., A. N. Garland y R. C. Janaway (Eds.), pp. 179-197. Manchester University Press, Manchester.

BROOKS, S., y J. SUCHEY, 1990. Skeletal age determination based on the os pubis: a comparison of the Acsádi-Nemeskéri and Suchey-Brooks methods. Human Evolution 5(3): 227-238.

BUIKSTRA, J., D. UBELAKER y D. A. AFTANDILIAN, 1994. Standards for data collection from human skeletal remains. Arkansas Archaeological Survey, Fayetteville.

COBO, B., 1956 [1893]. Historia del Nuevo Mundo. Ediciones Atlas, Madrid.

COHEN, J., 1960. A Coefficient of Agreement for Nominal Scales. Educational Psicology Measurement 20:37-46. 
COHEN, L., 1994. Old Age: Cultural and Critical Perspectives. Annual Review of Anthropology 23: 137-158.

CRAWFORD, S., 1999. Childhood in Anglo-Saxon England. Sutton, Stroud.

CHAKRABORTY, R., y P. P. MAJUMDER, 1982. On Bennet's measure of sex dimorphism. American Journal of Physical Anthropologist 59: 295-298.

CHAMBeRlAin, A., 2006. Demography in Archaeology. Cambridge University Press, Cambridge.

EARLE, T. K., 1991. Chiefdoms: power, economy and ideology. Cambridge University Press, Cambridge.

EMBER, C., 1975. Residential variation among hunter-gatherers. Behavior Science Research 3: 199-227. 1978. Myths about hunter-gatherers. Ethnology 17: 439-448.

FEREMBACH, D., I. SCHWIDETZKY y M. STLOUKAL, 1980. Recommendations for age and sex diagnoses of skeletons. Journal of Human Evolution 9: 517-549.

GALLARDO, F., M. URIBE y P. AYALA, 1995. Arquitectura inka y poder en el pukara de Turi, norte de Chile. Gaceta Arqueológica Andina III (2): 151-171.

GORDON, A., 1964. El método de excavación aplicado en el cementerio Pica-8. Boletín de la Sociedad de Amigos de la Arqueología de Santiago 2: 11-20.

GUAMAN POMA, F., 1615. Nueva Crónica y Buen Gobierno. Capítulo 10. El Capítulo de la Visita General o Censo. En http://www. kb.dk/permalink/2006/poma/220

GUTIÉRREZ, E. S., 1990. Una sociedad cacical agrícola: el sitio arqueológico Kaminaljuyú/San Jorge, Guatemala. Boletín de Antropología Americana 22: 123-144.

HAMILTON, W. D., 1971. Selection of selfish and altruistic behavior in some extreme models. En Man and Beast: Comparative Social Behavior, J. F. Eisenberg, y W. S. Dillon (Eds.), pp. 57-91. Smithsonian, Washington.

1972. Altruism and related phenomena, mainly in social insects. Annual Review of Ecology and Systematics 3: 193-232.

1975. Innate social aptitudes of man: an approach from evolutionary genetics. En Biosocial Anthropology, R. Fox (Ed.), pp. 133153. Malaby Press, London.
HARLOW, M., y R. LAURENCE, 2002. Growing Up and Growing Old in Ancient Rome. Routledge, London.

HARRISON, G. A., 1995. The Human Biology of the English Village. Oxford University Press, Oxford.

HAWKES, K., 2003. Grandmothers and the Evolution of Human Longevity. American Journal of Human Biology 15: 380-400.

HAWKES, K., J. O'CONNELL y N. BLURTON JONES, 1997. Ha$\mathrm{dza}$ Women's Time Allocation, Offspring Provisioning, and the Evolution of Long Postmenopausal Life Spans Current Anthropo$\log 38$ : 551-557.

HAWKES, K., J. O'CONNEL, N. BLURTON JONES, H. ÁLVAREZ y E. CHARNOV, 1998. Grandmothering, menopause, and the evolution of human life histories. Proceedings of the National Academy of Science 95: 1336-1339.

HEADLAND, T. N., 1989. Population decline in a Philippine Negrito hunter-gatherer society. American Journal of Human Biology 1: 59-72.

HILL, K., y A. M. HURTADO, 1995. Ache Life History. The Ecology and Demography of a Foraging People. Aldine de Gruyter, New York.

HOWELL, N., 1979. Demography of the Dobe! Kung. Academic Press, New York.

1982. Village composition implied by a paleodemographic life table: the Libben site. American Journal of Physical Anthropology 59: 263-270.

HOPPA, R. D., y J. W. VAUPEL, 2002. The Rostock Manifesto for Paleodemography: the way from stage to age. En Paleodemography. Age Distributions from Skeletal Samples, R. Hoppa y J. Vaupel (Eds.). Cambridge University Press, Cambridge.

IPIÑA, S., y A. I. DURAND, 2000. A measure of sexual dimorphism in populations which are univariate normal mixtures. Bulletin of Mathematical Biology 62: 925-941.

2004. Inferential assessment of the MI index of sexual dimorphism: A comparative study with some other sexual dimorphism measures. Bulletin of Mathematical Biology 66: 505-522.

JANETTA, A. B., y S. H. PRESTON, 1991. Two centuries of mortality change in central Japan: the evidence from a temple death register. Population Studies 45: 417-436.

JOSEPHSON, S., K. JUELL y A. ROGERS, 1996. Estimating sexual dimorphism by method of moments. American Journal of Physical Anthropology 100: 191-206. 
KEMKES-GROTTENHALER, A., 2006 Aging through the ages: historical perspectives on age indicator methods. En Paleodemography. Age Distributions from Skeletal Samples, R. Hoppa y J. Vaupel (Eds.). Cambridge University Press, Cambridge.

KLEPINGER, L., 2006. Fundamentals of Forensic Anthropology. Wiley and Sons, New Jersey.

LA FONTAINE, J. S., 1986. Sex and Age as Principles of Social Differentiation. Academic Press, London.

LAHDENPERÄ, M., V. LUMMAA, S. HELLE, M. TREMBLAY y A. RUSSELL, 2004. Fitness benefits of prolonged post-reproductive lifespan in women. Nature 428: 178-181.

LANDIS, J. R., y G. G. KOCH, 1977. The measurement of observer agreement for categorical data. Biometrics 33: 159-174.

LLAGOSTERA, A., 1976. Hipótesis sobre la expansión incaica en la vertiente occidental de los Andes Meridionales. En Homenaje al Dr. Gustavo Le Paige s.j., J. M. Cassassas (Ed.). Universidad del Norte, Antofagasta.

1995. San Pedro de Atacama: nodo de complementariedad reticular. En Integración Surandina: Cinco siglos después, X. Albó et al. (Eds.), pp. 17-39. Estudios y Debates Regionales Andinos 91, Centro de Estudios Regionales Andinos Bartolomé de las Casas, Corporación Norte Grande, Taller de Estudios Andinos, Universidad Católica del Norte, Cusco.

LOVEJOY, C., R. MEINDL, T. PRYZBECK, y R. P. MENSFOR$\mathrm{TH}, 1985$. Chronological metamorphosis of the auricular surface of the ilium: a new method for the determination of adult skeletal age at death. American Journal of Physical Anthropology 68: $15-28$

LUMBRERAS, L., 1974. La Arqueología como ciencia social. Ediciones Históricas, Editorial Nueva Educación, Lima.

MARINI, E., W. RACUGNO y S. BORGOGNINI, 1999 Univariate estimates of sexual dimorphism: the effects of intrasexual variability. American Journal of Physical Anthropology 109: 501-508.

MAYNARD, J., 1964. Group selection and kin selection. Nature 201: 1145-1147.

MEAD, M., 1970. Cultura y compromiso. El mensaje de la nueva generación. Gedisa Editorial, España.

MEINDL, R. S., C. O. LOVEJOY y R. P. MENSFORTH, 1983. Skeletal age at death: accuracy of determination and implications for paleodemography. Human Biology 55: 73-87.
MEINDL, R., C. LOVEJOY, R. MENSFORTH y R. WALKER, 1985. A revised method of age determination using the os pubis with a review and tests of accuracy of other current methods of pubic symphyseal aging. American Journal of Physical Anthropology 68: 29-45

MEINDL, R. S., y K. F. RUSSELL, 1998. Recent advances in method and theory in paleodemography. Annual Review of Anthropology 27:375-399.

MELLER, C., I. URZÚA, G. MONCADA y C. VON OHLE, 2009. Prevalence of oral pathologic findings in an ancient pre-Columbian archaeological site in the Atacama Desert. Caries Research 15: $287-294$.

MILLER, D., y C. TILLEY, 1984. Ideology, power and prehistory: an introduction. Ideology, Power and Prehistory. Cambridge University Press, Cambridge.

MORAGAS, C., 1995. Desarrollo de las comunidades prehispánicas del litoral Iquique-desembocadura río Loa. Hombre y Desierto 9 (I): $65-80$

MUNIZAGA, J., 1974. Paleopatología chilena (Informe preliminar). Antropología (Nueva Epoca) 1: 35-39.

MURDOK, G., 1967. The Ethnographic Atlas. University of Pittsburg Press, Pittsburg.

MURRA, J. V., 1972. El 'control vertical' de un máximo de pisos ecológicos en la economía de las sociedades andinas. En Visita a la provincia de León de Huánuco en 1562, Iñigo Ortiz de Zúñiga, J. V. Murra (Ed.), Huánuco.

1978. Los olleros del Inka: hacia una historia y arqueología del Qollasuyu. En Historia, problema y promesa. Homenaje a Jorge Basadre, vol. I, Lima.

1983 [1955]. La organización económica del Estado Inca. Editorial Siglo XXI, México.

MURRAY, K., y T. MURRAY, 1991. A test of the auricular surface aging technique. Journal of Forensic Sciences 36: 1162-1169.

NIELSEN, A., 1995. Architectural performance and the reproduction of social power. En ExpandingArchaeology, J. M. Skibo, W. H. Walker y A. Nielsen (Eds.). University of Utah Press, Salt Lake City.

2007. Armas significantes: tramas culturales, guerra y cambio social en el sur andino prehispánico. Boletín del Museo Chileno de Arte Precolombino 12 (1): 9-41. 
NUÑEZ, L., 1984. Tráfico de complementariedad de recursos entre las Tierras Altas y el Pacífico en el Aérea Centro Sur Andina. Tesis doctoral, Departamento de Antropología Cultural, Universidad de Tokio.

NÚÑEZ, L., y C. MORAGAS, 1983. Una ocupación con cerámica temprana en la secuencia del distrito de Cáñamo (costa desértica del norte de Chile): análisis y evaluación regional. Chungara 11: 31-61.

NÚÑEZ, L., y T. DILLEHAY, 1995 [1978]. Movilidad giratoria, armonía social y desarrollo en los Andes Meridionales: patrones de tráfico e interacción económica. Universidad Católica del Norte, Antofagasta.

OSBOURNE, D., T. SIMMONS y S. NAWROCKI, 2004. Reconsidering the auricular surface as an indicator of age at death. Journal of Forensic Sciences 49: 905-911.

PACHECO, A., 2010. Bioarqueología del conflicto: Lesiones esqueletales en la colección Pica 8 y su relación con situaciones de violencia interpersonal durante el período Intermedio Tardio (Región de Tarapacá, Chile). Memoria de título. Facultad de Ciencias Sociales, Universidad de Chile.

PACHECO, A., y R. RETAMAL, 2010. Posibles evidencias de conflicto en el Intermedio Tardío Tarapaqueño de Chile, reflexiones a partir de indicadores osteológicos y arqueológicos del cementerio Pica 8. Resumen. ХVII Congreso Nacional de ArqueologíaArgentina.

PHENICE, T. W., 1969. A newly developed visual method of sexing the os pubis. American Journal of Physical Anthropology 30: 297-301.

RETAMAL, R., yA. RIVERA, 2005 Ms. Afinidades biológicas y estatus en el cementerio Pica 8. Informe presentado para el proyecto FONDECYT 1030923. Manuscrito no publicado.

RETAMAL, R., y G. MANRÍQUEZ, 2006. Efectos de la deformación craneana intencional sobre la expresión del dimorfismo sexual en cráneos adultos: Un análisis morfométrico geométrico. Actas del XVII Congreso de Arqueología Chilena: 801-812.

RETAMAL, R., A. PACHECO y M. URIBE, 20o6. Perfil osteobiográfico del cementerio Pica 8: Paleopatología y modos de vida. Actas del XVII Congreso de Arqueología Chilena: 71-81.

RETAMAL, R., A. PACHECO y J. PINARES, 2009. Un caso de punta de proyectil incrustada en esqueleto de la colección Pica 8. Resumen. XVIII Congreso de Arqueología Chilena.

RILEY, M. W. 1984. Foreword. En Age and Anthropological Theory, D. I. Kertzer y Keith, J. (Eds.), pp. 7-10. Cornell University Press, Ithaca.
SANHUEZA, J., y O. OLMOS, 1985. Usamaya I, cementerio indígena en Isluga, altiplano de Iquique, I Región-Chile. Chungara 8: $200-204$.

SARKAR, N. K., 1951. A note on abridged life tables for Ceylon, 1900-1947. Population Studies 4: 439-443.

SCHAEFER, M., S. BLACK y L. SCHEUER, 2009. Juvenile Osteology: A Laboratory and Field Manual. Academic Press, London.

SCHIAPPACASSE, V., V. CASTRO y H. NIEMEYER, 1989. Los desarrollos regionales en el Norte Grande (1000-1400 DC). En Culturas de Chile. Prehistoria. Desde sus orígenes hasta los albores de la conquista, J. Hidalgo et al. (Eds.). Editorial Andrés Bello, Santiago.

SCOTT, S., y C. J. DUNCAN, 1998. Human Demography and Disease. Cambridge University Press, Cambridge.

SHAHAR, S., 1993. Who were old in the Middle Ages? Social History of Medicine 6: 313-341.

SHANKS, M., y I. HODDER. 1995. Processual, postprocessual and interpretive archaeologies. En Interpreting Archaeology. Finding meanings in the past. Routledge, Londres y Nueva York.

SMITH, T. E., 1960. The Cocos-Keeling Islands: a demographic laboratory. Population Studies 14: 94-130.

SPIRDUSO, W., 1995. Physical dimensions of aging. Human Kinetics, Champaign.

ST. HOYME, L.E., y M. Y. ISCAN, 1989. Determination of sex and race: accuracy and assumptions. En Reconstruction of life from the skeleton, M. Y. Iscan y K.A.R. Kennedy (Eds.), pp. 53-93. WileyLiss, New York.

SUTHERLAND, L. D., y J. M. SUCHEY 1991. Use of the ventral arc in pubic sex determination. Journal of Forensic Sciences 36:501-511.

SWISHER, C., G. CURTIS, T. JACOB, A. GETTY y A. SUPRIJO, 1994. Age of the earliest known hominids in Java, Indonesia. Science 263: 1118-1121.

TOMASSON, R. F., 1977. A millennium of misery: the demography of the Icelanders. Population Studies 31: 405-427.

URIBE, M., 1999-2000. La arqueología del Inka en Chile. Revista Chilena de Antropología 15: 63-97.

2006. Acerca de complejidad, desigualdad social y el complejo cultural Pica-Tarapacá en los Andes Centro-Sur (1000-1450 DC). Estudios Atacameños 31: 91-114. 
URIBE, M., y L. ADAN, 2004. Acerca del dominio inka, sin miedo, sin vergüenza. Chungara 36:467-480.

2005. Evolución social a través de la prehistoria tardía de Tarapacá (Norte Grande de Chile). Ponencia presentada en el Primer Taller de Teoría Arqueológica en Chile, Universidad de Chile, Santiago.

URZÚA, I., C. MELLER, G. MONCADA, A. PACHECO y R. RETAMAL, 2007. Oral health findings in a pre-Hispanic ancient culture of Chile. Caries Research 41: 298.

WALKER, P. L., J. R. JOHNSON y P. M. LAMBERT, 1988. Age and sex biases in the preservation of human skeletal remains. American Journal of Physical Anthropology 76: 183-188.
WALKER, A., y R. LEAKEY, 1993. The Nariokotome Homo erectus Skeleton. Harvard University Press, Cambridge.

WEISS, K. M., 1973. Demographic models for anthropology. AmericanAntiquity 38: 1-186.

WHITE, T., y P. FOLKENS, 2005. The Human Bone Manual. Elsevier Academic Press, London.

WILLIAMS, G. C., 1957. Pleiotropy, natural selection, and the evolution of senescence. Evolution 11: 389-411.

ZLATAR, V., 1984. Cementerio Prehispánico Pica-8. Universidad de Antofagasta, Antofagasta. 